\title{
Positive Regulation of Mitosis
}

National Cancer Institute

\section{Source}

National Cancer Institute. Positive Regulation of Mitosis. NCI Thesaurus. Code C40783.

Mitosis Induction involves the initiation of activities involved in division of the eukaryotic

cell nucleus to produce two daughter nuclei that contain the same chromosome and

DNA content as that of the parent cell. 